INPLASY

PROTOCOL

To cite: Cao et al. Chinese Herbal Medicine Duhuojisheng Decoction for Knee Osteoarthritis: A protocol for systematic review and metaanalysis. Inplasy protocol 2020120095. doi:

10.37766/inplasy2020.12.0095

Received: 18 December 2020

Published: 18 December 2020

Corresponding author: Ji-hui Cao

979752339@qq.com

Author Affiliation:

Chengdu University of

Traditional Chinese Medicine

Support: 2018JC018.

Review Stage at time of this submission: Data analysis.

Conflicts of interest: None.

\section{Chinese Herbal Medicine Duhuojisheng Decoction for Knee Osteoarthritis: A protocol for systematic review and meta-analysis}

Cao, JH1'; Feng, DG2; Wang, YZ3; Zhang, HY4; Zhao, YD5; Sun, ZH6; Feng, SG7; Chen, Y8; Zhu, MS 9 .

Review question / Objective: The purpose of this systematic review and meta-analysis is to evaluate the effectiveness and safety of DHJSD in the treatment of KOA.

Condition being studied: KOA is a common disease in orthopedics; it has the characteristics of high prevalence, wide range of lesions, and severe late dysfunction. Among people over 60 years old, the incidence of the disease is about $10 \%$ in men and $18 \%$ in women. The knee joint is the main body joint with the highest incidence of osteoarthritis. The main clinical manifestations of KOA are knee joint pain limited mobility, and a high disability rate, which has a serious impact on the quality of life of patients. With the aging of the global population, the incidence of the disease is increasing year by year. The trend has brought a huge economic burden to the society.

INPLASY registration number: This protocol was registered with the International Platform of Registered Systematic Review and Meta-Analysis Protocols (INPLASY) on 18 December 2020 and was last updated on 18 December 2020 (registration number INPLASY2020120095).

\section{INTRODUCTION}

Review question / Objective: The purpose of this systematic review and meta-analysis is to evaluate the effectiveness and safety of DHJSD in the treatment of KOA.

Rationale: As a classic prescription for treating knee osteoarthritis (KOA), Du-Huo-
Ji-Sheng-Decoction (DHJSD) has been widely recognized for its clinical efficacy. The purpose of this systematic review and meta-analysis is to evaluate the effectiveness and safety of DHJSD in the treatment of KOA.

Condition being studied: KOA is a common disease in orthopedics; it has the 
characteristics of high prevalence, wide range of lesions, and severe late dysfunction. Among people over 60 years old, the incidence of the disease is about $10 \%$ in men and $18 \%$ in women. The knee joint is the main body joint with the highest incidence of osteoarthritis. The main clinical manifestations of KOA are knee joint pain limited mobility, and a high disability rate, which has a serious impact on the quality of life of patients. With the aging of the global population, the incidence of the disease is increasing year by year. The trend has brought a huge economic burden to the society.

\section{METHODS}

Search strategy: The following databases will be searched: PubMed, Embase, Web of Science, Cochrane Library, Chinese Biomedical Medical Database, China National Knowledge Infrastructure, Chinese Science and Technology Periodical Database and Wanfang Database. We will search the records in these databases covering January 2011 to December 2020. Search terms consist of disease (knee osteoarthritis OR knee osteoarthritides OR knee joint osteoarthritis OR knee pain OR osteoarthritis of knee OR knee arthritis) and intervention (Du-huo-ji-shengDecoction OR Du-huo-ji-sheng-Tang) and research types (randomized controlled trial).

Participant or population: This review will consider all relevant studies DHJSD in the treatment of KOA. There are no restrictions of gender, race or age.

Intervention: The intervention method of the experimental group is DHJSD. There are no restrictions on administration time, frequency and dosage form. Research combined with other interventions (such as acupuncture, massage) will be excluded.

Comparator: Treatments other than DHJSD (eg, gui zhi ge gen Decoction, placebo, rehabilitation treatments). There are no restrictions on administration time, frequency and dosage form.
Study designs to be included: Randomized controlled trials.

Eligibility criteria: Interventions will include any type of Duhuo Jisheng Decoction for improvement of Symptoms of knee osteoarthritis.

Information sources: The following databases will be searched: PubMed, Embase, Web of Science, Cochrane Library, Chinese Biomedical Medical Database, China National Knowledge Infrastructure, Chinese Science and Technology Periodical Database and Wanfang Database.

Main outcome(s): The main results will be Hospital for Special Surgery (HSS) score.

Additional outcome(s): Secondary results will be visual analogue scale (VAS) scores and Western Ontario and McMaster Universities Osteoarthritis Index (WOMAC).

Data management: We will use EndNote X9 software to manage the records of searched electronic databases. The initial selection will involve scanning of the titles and abstracts of the retrieved studies. The full text of relevant studies will then be reviewed in accordance with the inclusion criteria by 2 authors (DGF and YZW). Potentially relevant articles will be reviewed independently by 2 authors to determine if they meet the criteria. Any disagreement between authors will be resolved by consensus with the third author (HYZ).

Quality assessment / Risk of bias analysis: The risk of bias in included studies will be assessed independently by 2 reviewers (YDZ and ZHS) using the Cochrane Risk of Bias Tool, with any disagreement resolved by consensus or by discussion with a third reviewer (MSZ). The tool has 7 domains, which includes random sequence generation, blinding of outcome assessment, blinding of participants and personnel, allocation concealment, selective reporting, incomplete outcome data, and other sources of bias. The risk of bias in each item is rated as "high", "low risk", or "unclear of bias". 
Strategy of data synthesis: In order to summarize the therapeutic effect of DHJSD on KOA, we will use Review Manager Version 5.3 (RevMan V5.3, Cochrane Center, Copenhagen, Denmark) to evaluate the difference between the intervention group and the control group in the study. The treatment effect will be assessed by the mean value. The difference of $95 \%$ confidence interval $(\mathrm{Cl})$ will be measured. In processing dichotomy data, we will consider the processing effect as the relative risk of $95 \% \mathrm{Cl}$; other binary data will be converted to RR. Then the $\mathrm{X} 2$ test will be used to determine whether there is heterogeneity between the studies (the test level is $a=0.1$ ). If $P>0.1$ or $I^{2} \leq 50 \%$, it is considered that there is no heterogeneity among multiple studies, and a fixed-effect model can be used to conduct a Metaanalysis of the combined effect size. If $P \leq 0.1$ or $I^{2}>50 \%$, it is considered that there is obvious heterogeneity between multiple studies, and random effects models can be used to combine effect size and analysis. If the heterogeneity is too large $\left(I^{2} \geq 75 \%\right)$, it will not be combined and only a descriptive analysis will be performed.

Subgroup analysis: If the number of included studies is sufficient, subgroup analysis will be conducted based on the patient's age, gender, treatment course, control group intervention methods, and dosage forms.

Sensibility analysis: In order to verify the stability of the primary outcomes, sensitivity analysis will be performed according to the study design, sample size, methodological quality, the effect of missing data of the included studies.

Language: Without any language or publication status restrictions.

Country(ies) involved: China.

Other relevant information: When data are missing, we will look for the reason. Then, we will contact the corresponding author to obtain and verify the data if possible. If this does not work, we will only analyze the available data.
Keywords: Du-Huo-Ji-Sheng-Decoction, systematic review, knee osteoarthritis, protocol.

Dissemination plans: The results of this systematic review will be published in a peer-reviewed journal or presented at conferences.

Contributions of each author:

Author 1 - Ji-hui Cao - The author drafted the manuscript.

Email: 979752339@qq.com

Author 2 - Da-gang Feng - The author provided statistical expertise.

Email: 847704614@qq.com

Author 3 - Yan-zhi Wang - The author contributed to the development of the selection criteria, and the risk of bias assessment strategy.

Email: 392811418@qq.com

Author 4 - Hai-yan Zhang - The author contributed to the development of the selection criteria, and the risk of bias assessment strategy.

Email: 386258394@qq.com

Author 5 - Yu-dong Zhao - The author ACTS as a supervisor.

Email: derfakir@stu.cdutcm.edu.cn

Author 6 - Zai-hui Sun - The author contributed to the development of the selection criteria, and the risk of bias assessment strategy.

Email: 408128933@qq.com

Author 7 - Shu-gui Feng - The author contributed to the development of the selection criteria, and the risk of bias assessment strategy.

Email: 1205343792@qq.com

Author 8 - Yi Chen - The author contributed to the development of the selection criteria, and the risk of bias assessment strategy.

Email: 893231832@qq.com

Author 9 - Ming-shuang Zhu - The author contributed to the development of the selection criteria, and the risk of bias assessment strategy.

Email: 3065720569@qq.com 